\title{
The known knowns and known unknowns of chronic pain
}

\author{
Philip Peng, MBBS
}

Received: 23 August 2015 / Accepted: 21 December 2015/Published online: 4 January 2016

(C) Canadian Anesthesiologists' Society 2016

“...there are known knowns; there are things we know we know. We also know there are known unknowns; that is to say we know there are some things we do not know. But there are also unknown unknowns - the ones we don't know we don't know."

Donald Rumsfeld, US Secretary of Defense, February 12, 2002

Chronic pain is costly - it is one of the known knowns. It affects the population widely and deeply. One in five Canadian adults suffers from chronic pain. ${ }^{1}$ In the United States, chronic pain affects 116 million adults, exceeding the number affected by heart disease, diabetes, and cancer combined. ${ }^{2}$ Globally, low back pain (LBP), neck pain, migraine, osteoarthritis, and other musculoskeletal disorders (MSD) are among the top 12 most common causes of disability in the Global Burden of Disease Study 2010. ${ }^{3}$ The overall annual socioeconomic burden of MSD, including LBP, in Canada was estimated to be $\$ 22.3$ billion in $2000-3 \%$ of Canada's gross domestic product (GDP). ${ }^{4}$ In the United States, chronic pain was associated with as much as US $\$ 635$ billion in healthcare costs and lost productivity in 2010. This is substantially more than the estimated costs for heart disease (309 billion), diabetes (188 billion), and cancer (243 billion) in America. ${ }^{2}$

P. Peng, MBBS ( $\square)$

Toronto Western Hospital, University Health Network, University of Toronto, 399 Bathurst Street, Toronto,

ON M5T 2S8, Canada

e-mail: Philip.peng@uhn.ca
In this issue of the Journal, Mann et al. add one small piece of "unknown" to the "knowns". In their postal survey, albeit with a response rate of only $19 \%$, they found that chronic pain was associated with increased visits to doctors, emergency departments, and other healthcare professionals (chiropractors, physiotherapists). ${ }^{5}$ While a lot of effort has been spent on exposing the problems associated with chronic pain, attention should also be devoted to the solutions. In this article, the author presents a snapshot of the "knowns" and "unknowns" of these efforts in pain management.

Finding funding for resources to curb chronic pain is a challenge. In Canada, the healthcare expenditure rose from $7 \%$ of GDP in 1975 to $11 \%$ in $2014 .^{6}$ The healthcare expenditure cannot expand indefinitely, and it has been kept fairly consistent at less than $12 \%$ of GDP in the last five years. ${ }^{6}$ Resources available to help millions of Canadians with chronic pain are stretched thin. To make the situation worse, there are unknown unknowns regarding the resources (political and economic uncertainty) and the known social factors that increase the prevalence of chronic pain (i.e., aging of the population, rising prevalence of obesity, and progress in saving the lives of people with catastrophic injuries). ${ }^{2}$ All these factors oblige us to search for cost-efficient and costsaving strategies in pain management.

Provision of pain management can be accomplished at four fronts: patients (self-management), primary care providers (family physicians, chiropractors, physiotherapists), specialists (pain specialists, neurologists, specialists in physical medicine and rehabilitation), or pain clinics (ranging from modality-oriented clinics to interdisciplinary pain clinics).

Self-management refers to the individual's ability to manage the symptoms, treatment, physical and social 
consequences, and lifestyle changes inherent in living with a chronic condition. It is an important first step and constant companion to pain patients in their pain journey. The five core self-management skills are problem solving, taking action for change, decision-making, partnership with healthcare professionals, and using resources. ${ }^{7} \mathrm{~A}$ meta-analysis of self-management education programs for arthritis revealed a small but statistically significant reduction in both pain ratings and reports of disability. ${ }^{8}$ This modality can be effective on its own or in combination with treatment directed by a health professional. A chronic pain self-management program is a low-cost, easily accessible, patient-centred approach which is complementary to other pain treatments and should be more widely adopted as an important modality in pain management. ${ }^{9}$

Primary care is the platform where a pain patient first reports pain to the healthcare system and where the healthcare provider has the opportunity to provide timely relief and prevent acute pain from progressing to a persistent state. Primary care physicians are responsible for the care of $52 \%$ of chronic pain patients in the United States and $70 \%$ in Europe. ${ }^{10,11}$ Although many primary care practitioners are proficient in providing exemplary pain care, this is not a uniform experience. A number of barriers impede optimal pain care within the primary care system, including provider attitudes, lack of physician training (less than four hours of chronic pain training in their two-year residencies in Canada), patient cultural attitudes, access to treatment (drugs, physiotherapy, psychologist), geographical barriers, regulatory limitation (lack of national or regional networking of medical records or prescriptions), and so on. ${ }^{2}$ Primary care is the platform where most pain patients receive care; therefore, strategies need to be developed to overcome the barriers. One such example is ECHO (Extension for Community Healthcare Outcomes) Ontario Chronic Pain \& Opioid Stewardship, a project funded by the Ontario Ministry of Health and LongTerm Care addressing the problem of chronic pain management in Ontario. The goal of ECHO Ontario is to increase primary care providers' competence and confidence in managing chronic pain through telehealth technology. ${ }^{12}$ The geographic barriers are overcome by connecting primary care providers from all across Ontario via multipoint video and teleconference technology. Short didactic presentations and case-based learning are provided to bridge the identified knowledge gaps. Another such example is the Atlantic Mentorship Network for Pain and Addiction funded by two provincial governments (Nova Scotia and Newfoundland and Labrador). ${ }^{13}$ It is the largest mentor-mentee network designed to assist practitioners, especially those in primary care, in the management of persons with pain and addiction. To reflect the important role of primary care providers as gatekeepers ensuring that the right patient receives the right intervention, Canadian Institutes of Health Research has proposed funding opportunities to support Canada's Strategy for PatientOriented Research (SPOR) networks. The objective of SPOR is to foster evidence-informed healthcare by bringing innovative, diagnostic, and therapeutic approaches to the point of care so as to ensure greater quality, accountability, and accessibility of care. ${ }^{14}$ In the context of pain management, this means ensuring that the right pain patient receives the right pain care. Although the result of this funding will not be known for another year, two of the top six finalists for this funding are related to pain and musculoskeletal health.

Pain specialists are the third front in this battle against chronic pain. They are equipped with the knowledge, skills, and attitudes to care for individuals with pain. Moreover, they have expertise in the prevention, evaluation, diagnosis, treatment, and rehabilitation of patients with acute pain and chronic (cancer and non-cancer) pain. ${ }^{15}$ Pain specialists are instrumental in managing complex and challenging patients in a biopsychosocial model, and they can serve as leaders in pain management infrastructure. Up to now, the exact number of pain specialists in Canada is unknown, but it is likely a small number. In 2009, the number of physicians in the United States who were board certified for pain care was less than 3,500, and this number signified that there were 33,000 chronic pain patients for every pain specialist. ${ }^{2}$ The key issue in this third front is how to define a pain specialist. In 2005, the Canadian Academy of Pain Management (CAPM) was established for credentialing and improving pain education -similar to the mandate of the American Academy of Pain Medicine. Nevertheless, CAPM is only a professional and not a regulatory body. The certification does not mandate a scrupulous assessment of training. In 2010, the Royal College of Physicians and Surgeons of Canada (RCPSC) officially recognized Pain Medicine as a subspecialty that requires multidisciplinary training over two years. ${ }^{15}$ The first pain residency commenced in 2014, and the first RCPSC subspecialty exam in pain medicine will take place in the fall of 2016. Specialists currently practicing pain medicine can obtain RCPSC certification through the practice eligibility route. ${ }^{16}$ Although pain specialists will not be able to absorb all the workload for pain management, the formal recognition, licensure, and training of these specialists will be a win-win situation for future pain care. 
Because of the deleterious consequences of chronic pain on the patient's psychosocial and physical functioning, a multidisciplinary team approach is considered the optimal treatment paradigm by expert bodies such as the International Association for the Study of Pain. The effectiveness of this approach is evidence based; ${ }^{2,17}$ however, resources are scarce and access is limited. A comprehensive study on multidisciplinary clinics in Canada estimated that one multidisciplinary pain facility served an average of 258,000 Canadians and wait times were long (months to years). ${ }^{18}$ Not every patient with chronic pain requires a multidisciplinary approach. More work is needed to identify that the right patient is getting the right care and to develop a system that facilitates allocating patients to the right place.

Resources are always used best for prevention and education. Indeed, one of the best known knowns is "an ounce of prevention is worth a pound of cure" (Benjamin Franklin). Resources for curbing obesity, preventing injury, aggressively treating acute pain, and promoting the use of certain vaccines (e.g., for herpes zoster) are likely to be more cost-effective than treating chronic pain that develops as a result of poor prevention. This requires a colossal and coordinated effort from the federal and provincial governments. Nevertheless, the lesser-known known is that preventive efforts are less likely to draw votes for politicians than treatment efforts (e.g., increase in patient access to magnetic resonance imaging, surgeries, or treatment), as the results from prevention are not usually apparent within politicians' terms of office (election cycles). Thus, pain education is not just for healthcare providers and patients but also for politicians, the public, and administrators. ${ }^{19}$ Without pressure from voters, vision from politicians, and insightful thinking from administrators, pain care will still be fragmented, barriers in primary care will still exist, and resources will still be misspent.

A physician has three responsibilities. The first is to manage patients who have disease, and the second is to manage disease of patients. The third responsibility is to manage the system where patients and disease exist. Historically, the last responsibility plays a substantial role in relieving human suffering and reducing mortality (infection control, vaccine, hygiene improvement, injury and disease prevention, developing more efficient systems for delivery of care). The known knowns are that chronic pain will remain costly and the resource will continue to be limited. The known unknowns are how we embark on developing a more efficient system for best use of resources and how we leverage political power to achieve this objective. We need leaders in pain care, not just new treatments or new drugs, to help us develop a resourceefficient system in pain care.

\section{La douleur chronique: les choses que nous savons que nous savons et celles que nous savons que nous ne savons pas}

«...il y a des choses, nous savons que nous les savons. Nous savons aussi que nous en ignorons. C'est-à-dire, nous savons qu'il y a des choses que nous ne savons pas. Mais il y a aussi des inconnues. Celles dont nous ne savons pas qu'elles nous sont inconnues. "

Donald Rumsfeld, Secrétaire à la Défense des États-Unis, 12 février 2002

La douleur chronique coûte cher - voilà l'une des choses que nous savons. Elle affecte la population dans son ensemble et profondément. En effet, un adulte canadien sur cinq souffre de douleur chronique. ${ }^{1}$ Aux États-Unis, elle touche 116 millions d'adultes, soit plus que le nombre total de personnes affectées par des cardiopathies, un diabète ou un cancer. $^{2}$ À l'échelle mondiale lombalgies, douleurs cervicales, migraines, ostéo-arthrite et autres troubles musculosquelettiques (TMS) comptent parmi les 12 causes les plus fréquentes d'invalidité citées dans l'étude portant sur le fardeau international de la maladie, publiée en 2010, « Global Burden of Disease Study $2010 »^{3}$ Le fardeau socioéconomique annuel global des TMS, incluant les lombalgies, était estimé à 22,3 milliards \$ en 2000 au Canada, soit $3 \%$ du produit national brut (PNB). ${ }^{4}$ Aux États-Unis, en 2010, la douleur chronique a été associée à un montant avoisinant les 635 milliards USD en coûts de soins de santé et en perte de productivité. Ces montants sont considérablement plus élevés que les coûts combinés estimés des cardiopathies (309 milliards), du diabète (188 milliards) et du cancer (243 milliards) en Amérique. ${ }^{2}$

Dans ce numéro du Journal, Mann et coll. ajoutent une chose que nous ne savions pas aux choses que nous savons. À partir de leur sondage par correspondance, qui n'a certes eu qu'un faible taux de réponse de $19 \%$, les auteurs ont pu déduire que la douleur chronique était associée à une augmentation des visites chez le médecin, à l'urgence et chez d'autres professionnels de la santé (chiropraticiens, physiothérapeutes). ${ }^{5}$ Bien que des efforts considérables aient été déployés pour faire la lumière sur les problèmes associés à la douleur chronique, il serait également utile de se pencher sur les solutions. Dans cet 
article, l'auteur présente un instantané des choses que nous savons et de celles que nous ignorons à propos de ces efforts en matière de prise en charge de la douleur.

La recherche de fonds pour financer les ressources permettant de juguler la douleur chronique est un défi. Au Canada, les dépenses en soins de santé sont passées de $7 \%$ du PNB en 1975 à $11 \%$ en $2014 .{ }^{6}$ Ces dépenses ne peuvent augmenter indéfiniment, et elles se sont maintenues relativement stables, soit en-deçà de $12 \%$ du PNB, au cours des cinq dernières années. ${ }^{6}$ Les ressources disponibles pour aider les millions de Canadiens souffrant de douleur chronique sont déjà utilisées au maximum de leur capacité. Pire encore, il existe des inconnues que nous ne connaissons pas concernant les ressources (l'incertitude politique et économique) et les facteurs sociaux connus qui augmentent la prévalence de la douleur chronique (c.-à-d. le vieillissement de la population, l'augmentation de l'obésité, et les progrès en matière de survie des personnes ayant subi des lésions catastrophiques). ${ }^{2}$ Tous ces facteurs nous poussent à trouver des stratégies de prise en charge de la douleur qui soient à la fois rentables et économiques.

La fourniture de services de prise en charge de la douleur peut se faire sur quatre fronts : par les patients (par autogestion), par les fournisseurs de soins de première ligne (omnipraticiens, chiropraticiens, physiothérapeutes), par des spécialistes (spécialistes de la douleur, neurologues, spécialistes en rééducation et réadaptation fonctionnelles), ou par les cliniques de la douleur (qu'il s'agisse de cliniques orientées vers les modalités ou de cliniques interdisciplinaires de prise en charge de la douleur).

Par autogestion, on fait référence à la capacité d'une personne de gérer ses symptômes, son traitement, les conséquences physiques et sociales ainsi que les changements de style de vie qui vont de pair avec une maladie chronique. Il s'agit d'une première étape importante qui accompagnera les patients atteints de douleur tout au long de leur voyage douloureux. Les cinq compétences clés de l'autogestion sont : la résolution de problèmes, l'action pour faire changer les choses, la prise de décision, le partenariat avec les professionnels de la santé et l'utilisation des ressources. ${ }^{7}$ Une méta-analyse s'intéressant aux programmes de formation en autogestion pour l'arthrite a révélé une réduction, petite certes mais significative d'un point de vue statistique, des scores de douleur et des rapports d'invalidité. ${ }^{8}$ Cette modalité peut être efficace seule ou combinée à un traitement dirigé par un professionnel de la santé. Un programme d'autogestion de la douleur chronique est une approche peu dispendieuse, facile d'accès et centrée sur le patient. Elle est complémentaire à d'autres traitements de la douleur; c'est pourquoi ce type de programme devrait être adopté plus globalement, étant donné qu'il est considéré comme une modalité importante dans la prise en charge de la douleur. ${ }^{9}$

Les soins de première ligne sont la plateforme où un patient de douleur rapporte, pour la première fois, sa douleur au système de santé. C'est là que le fournisseur de soins de santé a la possibilité d'offrir un soulagement opportun et d'empêcher une douleur aiguë de devenir persistante ou chronique. Les médecins de première ligne prennent soin de $52 \%$ des patients atteints de douleur chronique aux États-Unis, et de $70 \%$ en Europe. ${ }^{10,11}$ Bien que bon nombre de ces médecins sachent comment procurer des soins de la douleur exemplaires, leur expérience n'est pas uniforme. Plusieurs obstacles entravent la fourniture de soins de douleur optimaux dans le système de soins de première ligne, notamment l'attitude des fournisseurs, le manque de formation des médecins (moins de quatre heures de formation en douleur chronique au cours de leur résidence de deux ans au Canada), les attitudes culturelles des patients, l'accès aux traitements (médicaments, physiothérapie, consultations psychologiques), des obstacles géographiques, les limites réglementaires (absence d'une mise en réseau nationale ou régionale des dossiers médicaux ou des prescriptions), etc. ${ }^{2}$ Les soins de première ligne sont l'endroit où la plupart des patients en douleur se font soigner, c'est pourquoi il est crucial de mettre au point des stratégies qui permettront de surmonter les obstacles. Le projet ECHO (Extension for Community Healthcare Outcomes - Extension pour les mesures de soins de santé dans la collectivité) en est un bon exemple. Il s'agit d'un projet ontarien de prise en charge de la douleur chronique et des opioïdes, financé par le Ministère de la santé et des soins de longue durée de l'Ontario. L'objectif du projet ECHO Ontario est d'augmenter la compétence et la confiance des fournisseurs de soins de santé de première ligne en prise en charge de la douleur chronique via des technologies de télésanté. ${ }^{12}$ Les obstacles géographiques sont surmontés en connectant des fournisseurs de soins de santé de première ligne des quatre coins de la province grâce à des technologies de visioconférence et de téléconférence multipoint. De courtes présentations didactiques et l'apprentissage par cas sont proposés afin de combler les lacunes dans leurs connaissances. Un autre exemple a été financé par deux gouvernements provinciaux, la Nouvelle-Écosse et Terre-Neuve-et-Labrador; il s'agit du Atlantic Mentorship Network for Pain and Addiction (Réseau de mentorat atlantique pour la douleur et l'accoutumance). ${ }^{13}$ Il s'agit du plus important réseau de mentor - mentoré conçu pour aider les praticiens, et plus particulièrement ceux œuvrant dans les soins de première ligne, dans la prise en charge des personnes atteintes de douleur ou souffrant d'accoutumance. Afin de refléter le rôle crucial des fournisseurs de soins de santé de première 
ligne en tant que gardiens s'assurant que le bon patient bénéficie de la bonne intervention, les Instituts de recherche en santé du Canada ont offert des possibilités de financement pour soutenir les réseaux canadiens de Stratégie de recherche axée sur le patient (SRAP). L'objectif de la SRAP consiste à se doter de soins de santé fondés sur des données probantes en instaurant des méthodes diagnostiques et thérapeutiques innovatrices au lieu d'intervention, afin d'améliorer la qualité et l'accessibilité des soins, ainsi que la reddition de compte. ${ }^{14}$ Dans le contexte de la prise en charge de la douleur, cela signifie que le bon patient de douleur reçoit les bons soins pour sa douleur. Bien que le résultat de ce financement ne sera pas connu avant un an, deux des six finalistes de ce financement travaillent dans le domaine de la douleur et de la douleur musculosquelettique.

Les spécialistes de la douleur sont le troisième front de cette bataille contre la douleur chronique. Ils possèdent les connaissances, les compétences et les attitudes nécessaires pour prendre soin des personnes atteintes de douleur. De plus, ils sont des experts de la prévention, de l'évaluation, du diagnostic, du traitement et de la réadaptation de patients souffrant de douleur aiguë et chronique (cancéreuse et non cancéreuse). ${ }^{15}$ Les spécialistes de la douleur jouent un rôle primordial dans la prise en charge de patients complexes dans un modèle bio-psycho-social, et ils peuvent devenir des chefs de file dans l'infrastructure de prise en charge de la douleur. À ce jour, le nombre exact de spécialistes de la douleur au Canada est inconnu, mais il est probablement très faible. En 2009, le nombre de médecins aux États-Unis accrédités pour prodiguer des soins de la douleur était inférieur à 3500, ce qui signifie qu'il y avait 33000 patients de douleur chronique par spécialiste de la douleur. ${ }^{2}$ Le problème clé de ce troisième front est la façon dont on définit un spécialiste de la douleur. En 2005, la CAPM (Canadian Academy of Pain Management) a été créée afin d'établir une liste des qualifications et d'améliorer la formation en douleur - un mandat semblable à celui de l'American Academy of Pain Medicine. Toutefois, la CAPM est un organisme professionnel et non un organisme de réglementation. La certification ne requiert pas d'évaluation scrupuleuse de la formation. En 2010, le Collège royal des médecins et chirurgiens du Canada (CRMCC) a officiellement reconnu la médecine de la douleur comme une surspécialité nécessitant une formation pluridisciplinaire sur deux ans. ${ }^{15}$ Le premier programme de résidence en médecine de la douleur a débuté en 2014, et le premier examen de surspécialité du CRMCC dans ce champ de compétences se tiendra à l'automne 2016. Les spécialistes pratiquant présentement la médecine de la douleur peuvent obtenir une accréditation du CRMCC en suivant la Route d'évaluation par la pratique. ${ }^{16}$ Bien que les spécialistes de la douleur ne pourront pas absorber toute la charge de travail pour la prise en charge de la douleur, la reconnaissance formelle, l'attribution du droit d'exercice et la formation de ces spécialistes seront bénéfiques sur tous les plans pour les soins futurs en douleur.

En raison des conséquences délétères de la douleur chronique sur le fonctionnement psychosocial et physique du patient, une approche multidisciplinaire est considérée comme le nec plus ultra du traitement par les organismes spécialisés tels que l'IASP (International Association for the Study of Pain). L'efficacité de cette approche se fonde sur des données probantes; ${ }^{2,17}$ toutefois, les ressources sont rares et l'accès est limité. Une étude exhaustive des cliniques multidisciplinaires au Canada a estimé qu'un établissement de prise en charge multidisciplinaire de la douleur desservait en moyenne 258000 Canadiens et que les temps d'attente étaient longs (des mois, voire des années). ${ }^{18}$ Tous les patients souffrant de douleur chronique n'ont probablement pas besoin d'une approche multidisciplinaire. Des recherches supplémentaires sont de mise afin de déterminer que le bon patient reçoit les bons soins et de mettre au point un système qui facilite l'allocation des patients au bon endroit.

La prévention et la formation sont toujours les meilleurs endroits pour utiliser nos ressources. En effet, l'une des choses que nous savons le mieux est qu' « une once de prévention vaut une livre de guérison» (Benjamin Franklin), ou «Mieux vaut prévenir que guérir ». Les ressources servant à freiner l'épidémie d'obésité, prévenir les blessures, traiter de façon vigoureuse la douleur aiguë et promouvoir l'utilisation de certains vaccins (par ex., contre le zona) seront probablement plus payantes que celles allouées au traitement de la douleur chronique, qui survient lorsque la prévention n'est pas à la hauteur. Ceci nécessite des efforts colossaux et coordonnés des gouvernements fédéral et provinciaux. Toutefois, ce que nous savons moins, c'est que les efforts de prévention sont moins populaires auprès des politiciens que les efforts de traitement (par ex., l'augmentation de l'accès des patients à l'imagerie par résonance magnétique, aux chirurgies ou aux traitements), étant donné que les résultats de mesures de prévention sont en général moins tangibles pendant le mandat des politiciens (en raison des cycles d'élection). Dès lors, la formation en douleur ne devrait pas s'adresser uniquement aux fournisseurs de soins de santé et aux patients, mais aussi aux politiciens, au grand public et aux administrateurs. ${ }^{19}$ Sans pression des électeurs, sans vision des politiciens, et sans réflexion des administrateurs, les soins de douleur demeureront fragmentés, des obstacles seront toujours présents dans les soins de première ligne, et les ressources seront toujours mal allouées.

Un médecin a trois responsabilités. La première est de prendre en charge les patients atteints de maladies; la 
deuxième est de prendre en charge les maladies des patients. Sa troisième responsabilité est de prendre en charge le système dans lequel les patients et les maladies existent. Traditionnellement, cette dernière responsabilité joue un rôle prépondérant dans le soulagement de la souffrance humaine et la réduction de la mortalité (citons par exemple le contrôle des infections, les vaccins, l'amélioration de l'hygiène, la prévention des blessures et des maladies, ou encore la mise au point de systèmes plus efficaces pour la fourniture des soins). Ce que nous savons, c'est que la douleur chronique restera dispendieuse et que les ressources resteront limitées. Ce que nous savons que nous ignorons, c'est comment nous commencerons à mettre au point un système plus efficace pour optimiser l'utilisation des ressources et comment nous ferons pression sur le pouvoir politique pour atteindre cet objectif. Nous avons besoin de chefs de file en soins de la douleur, pas seulement de nouveaux traitements ou de nouveaux médicaments, pour nous aider à mettre au point un système rentable et efficace du point de vue de nos ressources en soins de la douleur.

Conflict of interest None declared.

Conflit d'intérêt Aucun.

\section{References}

1. Schopflocher D, Taenzer P, Jovey $R$. The prevalence of chronic pain in Canada. Pain Res Manag 2011; 16: 445-50.

2. Institute of Medicine of the National Academies. Relieving Pain in America: A Blueprint for Transforming Prevention, Care, Education and Research. Washington DC, Institute of Medicine, National Academies Press; 2011.

3. Vos T, Flaxman AD, Naghavi M, et al. Years lived with disability (YLDs) for 1160 sequelae of 289 diseases and injuries 19902010: a systematic analysis for the Global Burden of Disease Study 2010. Lancet 2012; 380: 2163-96.

4. Public Health Agency of Canada. Life with Arthritis in Canada A Personal and Public Health Challenge - 2010. Available from URL: http://www.phac-aspc.gc.ca/cd-mc/arthritis-arthrite/lwaicvaaac-10/pdf/arthritis-2010-eng.pdf (accessed October 2015).

5. Mann EG, Johnson A, VanDenKerkhof EG. Frequency and characteristics of healthcare visits associated with chronic pain: results from a population-based Canadian study. Can J Anesth 2016; 63: DOI:10.1007/s12630-015-0578-6.

6. Canadian Institute for Health Information. National Health Expenditure Trends, 1975 to 2014. Report - October 2014. Available from URL: http://www.cihi.ca/web/resource/en/nhex 2014_report_en.pdf (accessed October 2015).

7. McGillion M, LeFort SM, Webber K, Stinson JN. Pain selfmanagement: theory and process for clinicians. In: Lynch ME, Craig KD, Peng PW, editors. Clinical Pain Management - A Practical Guide. 1st ed. Oxford: Wiley-Blackwell Publisher; 2011. p. 193-9.

8. Warsi A, LaValley MP, Wang PS, Avorn J, Solomon DH. Arthritis self-management education programs: a meta-analysis of the effect on pain and disability. Arthritis Rheum 2003; 48: 2207-13.

9. Kroenke K, Bair MJ, Damush TM, et al. Optimized antidepressant therapy and pain self-management in primary care patients with depression and musculoskeletal pain: a randomized controlled trial. JAMA 2009; 301: 2099-110.

10. Breuer B, Cruciani $R$, Portenoy $R K$. Pain management by primary care physicians, pain physicians, chiropractors, and acupuncturists: a national survey. South Med J 2010; 103: 73847.

11. Breivik H, Collett B, Ventafridda V, Cohen R, Gallacher D. Survey of chronic pain in Europe: prevalence, impact on daily life, and treatment. Eur J Pain 2006; 10: 287-333.

12. Dubin RE, Flannery J, Taenzer P, et al. ECHO Ontario chronic pain \& opioid stewardship: providing access and building capacity for primary care providers in underserviced, rural, and remote communities. Stud Health Technol Inform 2015; 209: 1522.

13. Atlantic Mentorship Network. Available from URL: http://www. atlanticmentorship.com (accessed October 2015).

14. Canadian Institutes of Health Research. Strategy for PatientOriented Research. Canadian Institutes of Health Research. Available from URL: http://www.cihr-irsc.gc.ca/e/41204.html (accessed October 2015).

15. Morley-Forster P, Karpinski J. Pain medicine-a new credential in Canada. Pain Med 2015; 16: 1038-44.

16. Royal College of Physicians and Surgeons of Canada. Pain Medicine Residency. Available from URL: http://fhs.mcmaster. ca/pain-medicine-residency (accessed October 2015).

17. Ospina $M$, Harstall $C$. Multidisciplinary pain programs for chronic pain: evidence from systematic reviews. Alberta Heritage Foundation for Medical Research - Health Technology Assessment, Alberta, Canada, 2003: 1-48. Available from URL: http://www.health.alberta.ca/documents/Chronic-Pain-Meeting2003.pdf (accessed October 2015).

18. Peng P, Choiniere $M$, Dion D, et al. Challenges in accessing multidisciplinary pain treatment facilities in Canada. Can $\mathbf{J}$ Anesth 2007; 54: 977-84.

19. Peng $P W$. Education in pain medicine: a three dimensional approach. Pain Manag 2012; 2: 101-3. 